\title{
Imitators of Cherenkov and scintillation light pulses based on fast LEDs
}

\section{Sultim Lubsandorzhiev ${ }^{1}$}

Institute for Nuclear Research of the Russian Academy of Sciences

Moscow, pr-t 60-letiya Oktyabrya 7a, Russia

E-mail:sultim@inbox.ru

\section{Bayarto Lubsandorzhiev}

Institute for Nuclear Research of the Russian Academy of Sciences Moscow, pr-t 60-letiya Oktyabrya 7a, Russia

E-mail: lubsand@rambler.ru

\section{Yevgeniy Vyatchin}

Institute for Nuclear Research of the Russian Academy of Sciences Moscow, pr-t 60-letiya Oktyabrya 7a, Russia

E-mail: lubsand@inr.ru

\section{Nikita Ushakov}

Institute for Nuclear Research of the Russian Academy of Sciences Moscow, pr-t 60-letiya Oktyabrya 7a, Russia

E-mail: nikita.ushakoff@gmail.com

\section{Dmitriy Voronin}

Institute for Nuclear Research of the Russian Academy of Sciences Moscow, pr-t 60-letiya Oktyabrya 7a, Russia

E-mail: dmitriy.voronin@gmail.com

\section{Andrey Sidorenkov}

Institute for Nuclear Research of the Russian Academy of Sciences Moscow, pr-t 60-letiya Oktyabrya 7a, Russia

E-mail: andreassx7@gmail.com

\section{Arslan Lukanov}

Institute for Nuclear Research of the Russian Academy of Sciences Moscow, pr-t 60-letiya Oktyabrya 7a, Russia

E-mail: arslanlukanov@yandex.ru

\section{Nima Lubsandorzhiev}

Institute for Nuclear Research of the Russian Academy of Sciences Moscow, pr-t 60-letiya Oktyabrya 7a, Russia

E-mail:nilubs@rambler.ru

\footnotetext{
${ }^{1}$ Speaker

(C) Copyright owned by the author(s) under the terms of the Creative Commons 
We present results of a number of developments of light sources imitating Cherenkov and scintillation light pulses for testing and calibration measurements for Cherenkov and scintillation detectors. The light sources developed for such applications are based on fast ultra bright light emitting diodes. The light sources electronic schemes and their performances are discussed.

36th International Cosmic Ray Conference -ICRC2019-

July 24th - August 1st, 2019

Madison, WI, U.S.A. 


\section{Introduction}

Cherenkov and scintillation detectors are widely used in high energy and astroparticle physics experiments. In such detectors Cherenkov and scintillation light pulses produced by ionizing particles are registered by photosensorss of different types: classical vacuum photomultipliers (PMTs), hybrid photodiodes (HPDs), silicon photomultipliers (SiPMs), photodiodes (PDs) and avalanche photodiodes (APDs). Operation conditions existing in Cherenkov and scintillation detectors put specific requirements for photosensors. To select proper photosensors and to test and calibrate them in such detectors it is convenient to use proper sources of light pulses imitating Cherenkov and scintillation light pulses as close as possible. Moreover it is of importance to have such sources to optimize detector electronics including front-end electronics, ASIC and FPGA.

\section{Light emitting diodes}

Ultra bright light emitting diodes (LEDs) are very good candidates for light pulses sources. Nowadays LEDs are ubiquitous, cheap and easily available. The advent of ultra bright blue InGaN/GaN LEDs in the early 1990s opened new era in designing of light sources. Presently LEDs cover full visible range of light spectra extending to UV (down to $245 \mathrm{~nm}$ and even shorter) and infrared (up to $1500 \mathrm{~nm}$ and further). A variety of some LEDs emission spectra is shown in Fig.1 [1].

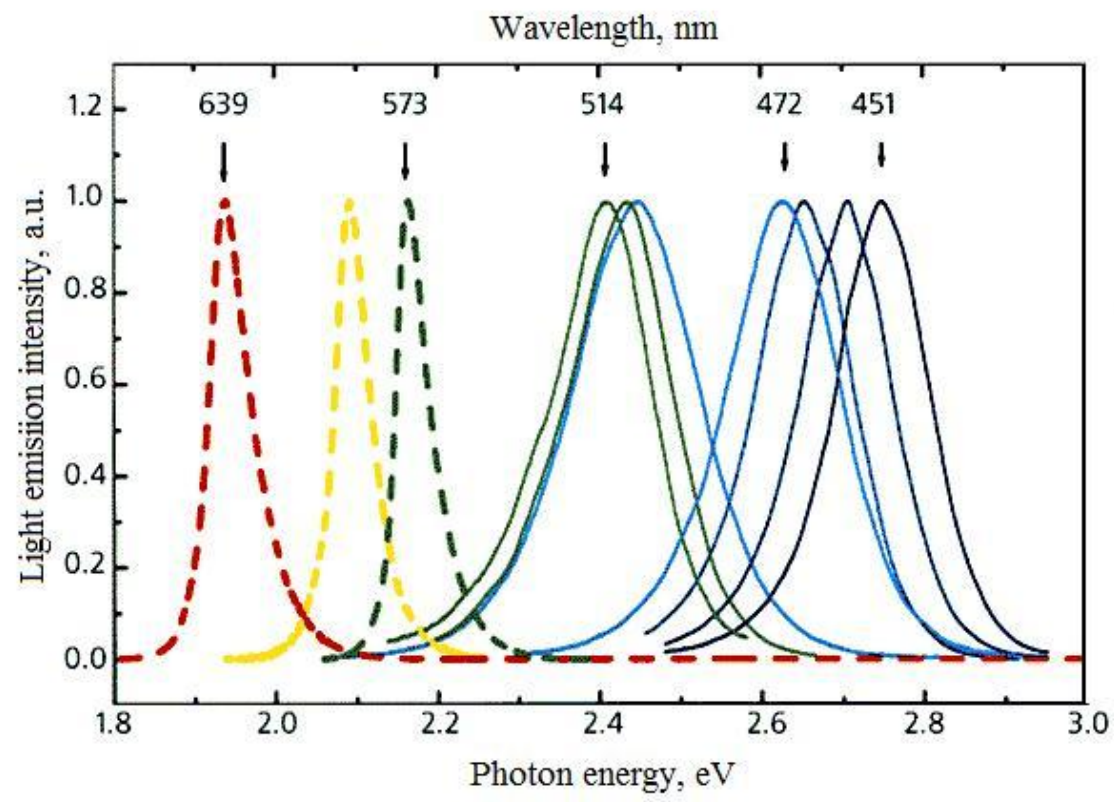

Fig.1. Emission spectra of some LEDs. 
Taking into account Cherenkov radiation spectrum and emission spectra of the most popular plastic and liquid scintillators the blue and near UV LEDs are of most importance for our purposes. The problem is to select LEDs by their emission kinetics and light yield.

Light emission kinetics of LEDs differs very much $[1,2]$ even among of LEDs of one type supplied by one manufacturer, from one sample of LED to another. LEDs are divided into two groups by their emission kinetics - "fast" and "slow". Light emission characteristics of LEDs depend also very much on their drivers - electronic schemes firing LEDs.

\section{LED drivers.}

All LED drivers themselves could be divided into two groups too. The first group is "low current" drivers. The maximum amplitude of current pulses for them is hundreds of mA. In Fig.2 a typical "low corrent" driver developed by J. Kapustinsky with colleagues [3] is shown.

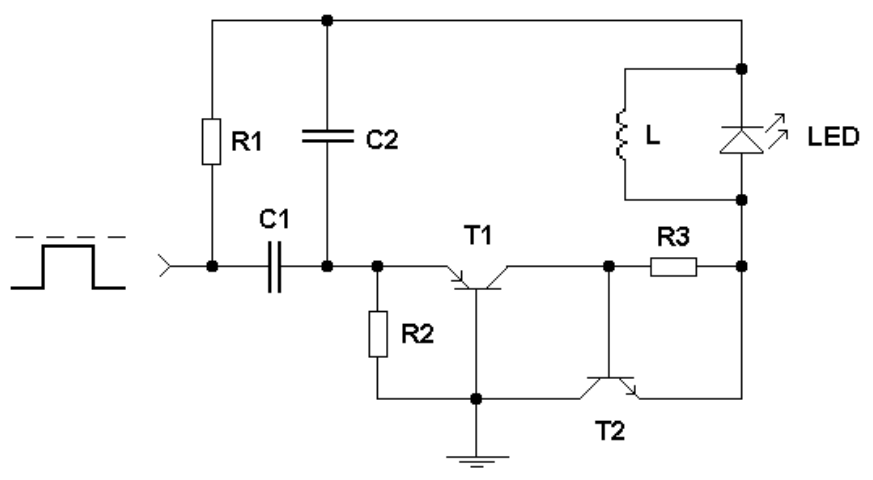

Fig.2. Fast "low current" LED driver, "Kapustinsky’s driver" [3], from [4].

The driver utilizes fast discharge of capacitor through complementary pair of fast "offshelves" transistors and fast LED. The light emission kinetics of the light source depends mostly on LED type. The fastest light pulses reached so far with the driver is $0.65 \mathrm{~ns}$ (FWHM) with a UV (365 nm) LED [4]. Usually with violet-blue (400-470 $\mathrm{nm}$ light emission maximum) fast LEDs one can get a few nanoseconds wide (1-3 ns) light pulses but with relatively low light yield. On the other hand the light yield may be quite enough for many applications, for example in the laboratory test measurements. Moreover we developed "low current" LED driver for field calibration measurements of Cherenkov arrays which provides up to $10^{8}$ photons per pulse light yield with less than $7 \mathrm{~ns}$ width (FWHM) [5].

The second group of drivers is so called "high current" drivers. With the drivers of the group the maximum amplitude of current pulses is 5-6 A and even more with a few nanosecond widths $[1,6]$. Typically for such kind of applications avalanche transistors drivers are used. An electrical scheme of typical driver of such type is presented in Fig.3. The drivers require relatively high voltage power supplies, typically several hundred Volts, $\sim 1 \mathrm{kV}$ in extreme cases [1]. Transistors for the drivers are either carefully selected from conventional bipolar transistors or special avalanche transistors [6]. 


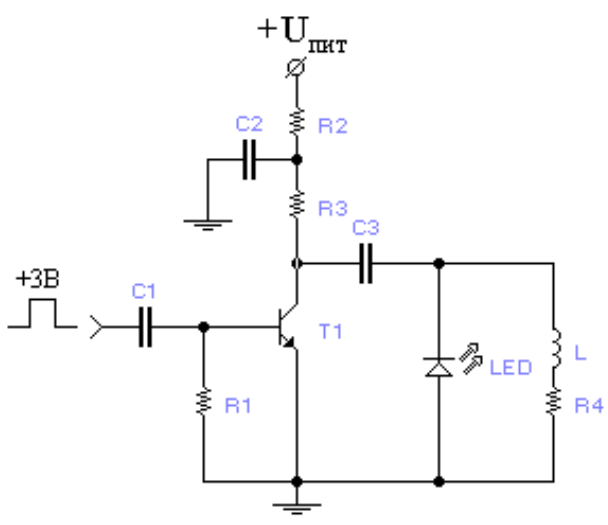

Fig. 3. Typical electrical scheme of "high current" LED driver, from [6]

As it was mentioned above the light emission kinetics of LEDs differs very much. Even LEDs from one batch of one type LEDs may have a variety of temporal behavior. As an example in Fig. 4 light emission kinetics of different samples of LEDs of the same type from the same manufacturer are shown. Here the light pulse waveforms were measured with the same one "high current" LED driver based on avalanche transistors. The driver provided $2.2 \mathrm{~A}$ amplitude current pulse with 1 ns width (FWHM).

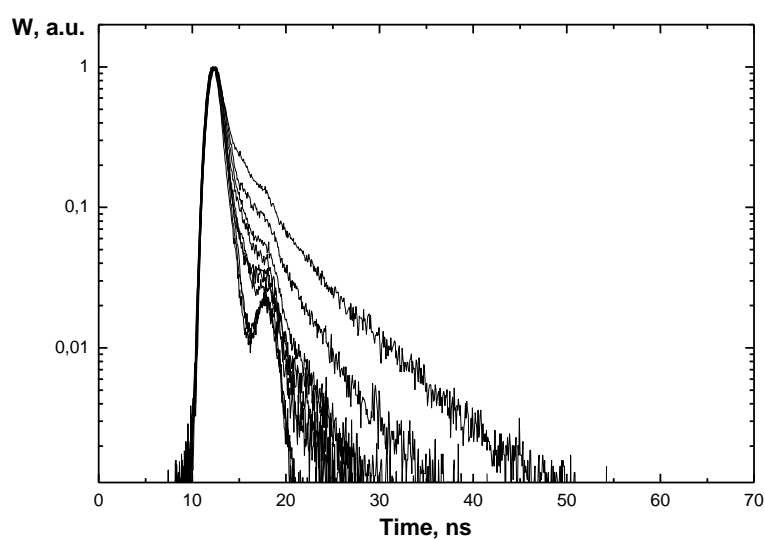

Fig. 4. Light emission kinetics of LEDs of the same type from GNR manufacturer.

In the time spectra shown in Fig. 4 some peaks at around $10 \mathrm{~ns}$ from the spectra's maximum are due to photoelectron backscattering [7] in PMT used in the measurements and have no relations to the LEDs light emission kinetics. The most part of LED whose temporal behavior shown in Fig. 4 belongs to very fast LEDs without tails. They can be used to imitate Cherenkov pulses. Their light pulse width is less than $1 \mathrm{~ns}$ (FWHM) and simultaneously the LEDs provides high light yield up to $10^{10}$ photons per pulse with "high current" drivers. The drivers with such LEDs can be used, for example, in calibration systems of multi-channel 
Cherenkov and scintillation detectors using either direct illumination of photosensors or distributed optical fibers system.

In Figs. 5 (left and right) light emission kinetics of "slow" and "very slow" LEDs fired by the same "high current" driver are presented.
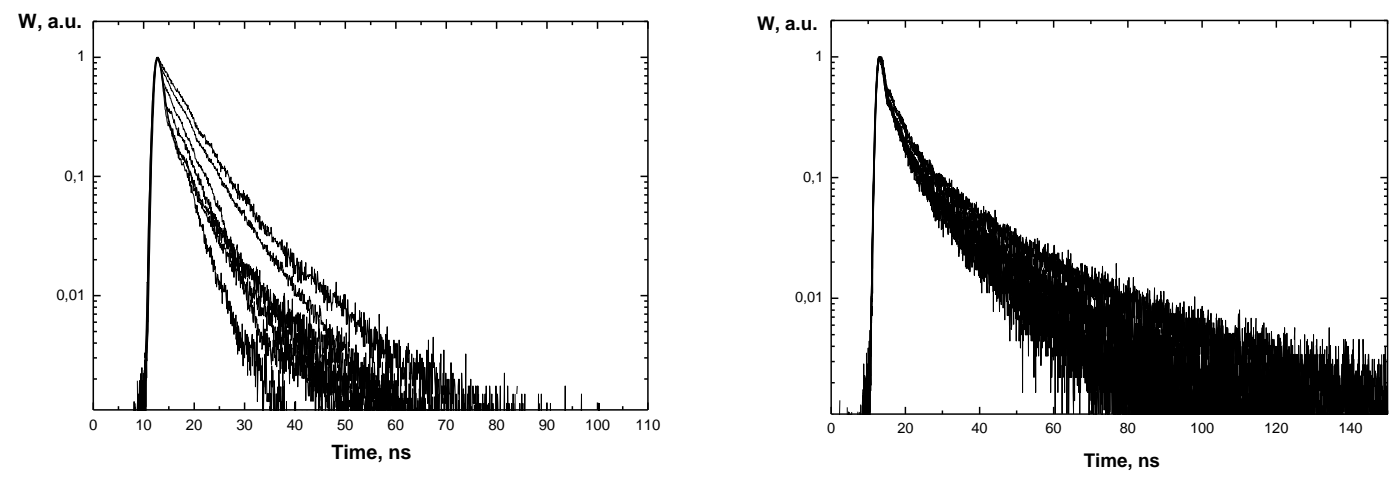

Fig. 5. Left - light emission kinetics of "slow" LEDs; Right - light emission kinetics of "very slow" LEDs. (All LEDs of different types).

From all those spectra it is quite clear that one can chose LEDs and proper drivers for many applications.

As an example light pulses waveform of polystyrene based plastic scintillator (actively used in high energy and astroparticle physics experiments) and light emission kinetics of LED equipped with "high current" driver are shown in Fig. 6. The line is the scintillator's waveform, the dots are the light emission kinetics of the LED. It should be noted that the light emission kinetics of the LED was the same with "low current" driver too. The LED has the same maximum $(\sim 430 \mathrm{~nm})$ of light emission spectrum as light emission spectrum of polystyrene based plastic scintillator $(\sim 30 \mathrm{~nm})$. The plastic scintillator was doped with PT as the first fluor and bis-MSB as the second WLS flour.

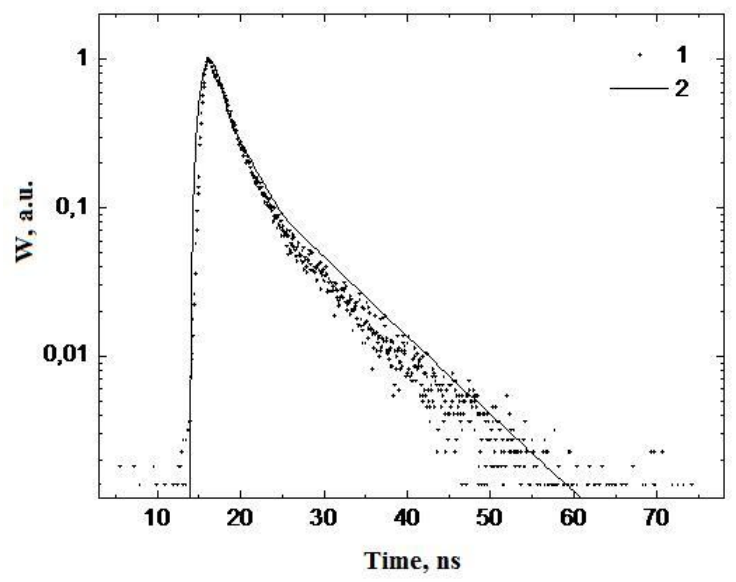

Fig. 6. Scintillation light pulse waveform of polystyrene based plastic scintillator (line) with $\lambda_{\max }=430 \mathrm{~nm}$ and light emission kinetics of ultra bright LED with $\lambda_{\max }=430 \mathrm{~nm}$. 
So, from a variety of light emission kinetics of LEDs it is practically always possible to find a proper LED sample with timing and amplitude parameters satisfying to the requirements of Cherenkov and scintillation detectors.

It should be noted that for Cherenkov detectors there are some peculiarities. Namely in case of single relativistic particle detection Cherenkov light pulses have very short - $\leq 1 \mathrm{~ns}$ width (FHWM). In case of extensive air shower (EAS) detection the Cherenkov pulse width is a few dozens of nanoseconds. Another case is Cherenkov light from high energy electromagnetic and hadronic showers produced in high energy interactions of e.g. neutrinos. Showers in this case are meters or tens of meters long, resulting in hundreds nanoseconds time width of Cherenkov light pulses with almost rectangular shape. To imitate Cherenkov light pulses due to such showers we developed special LED drivers and selected proper LED samples. The driver for such application is based on avalanche transistors like in "high current" drivers. To the transistor's collector a piece of coaxial cable is attached, see Fig. 7, its core end is free, the screen is grounded. Selected fast LED is switched to the transistor's emitter circuit. Adjustable capacitor (C2) and resistor (R2) are used to decrease light pulses rise and fall times and to match to the impedance of cable switched to the transistor's collector. In this case the cable's parasiric capacitor serves as a discharging capacitor providing LED with current pulse. The length of the cable defines light pulses width of the light source by a simple formula:

$$
\Delta \mathrm{t}=10 \cdot \mathrm{L}
$$

here $\Delta \mathrm{t}$ - light pulse's width in nanoseconds, $\mathrm{L}$ is the cable's length in meters.

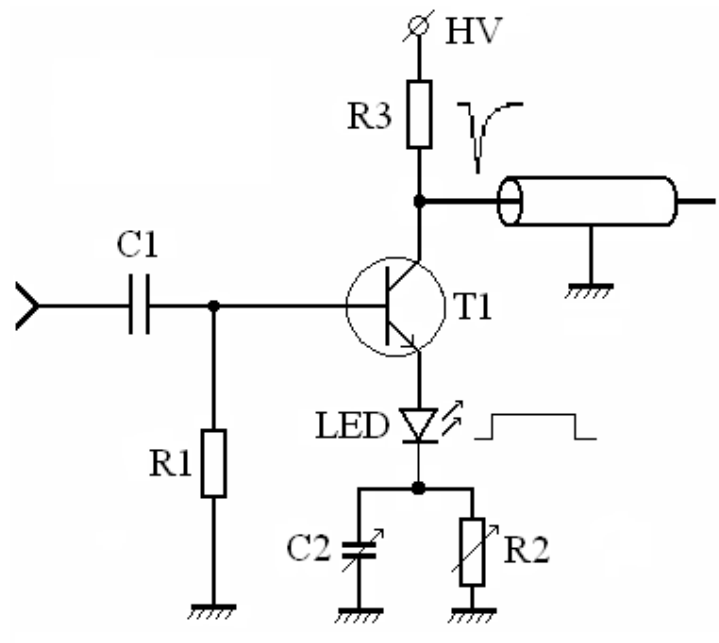

Fig. 7. Imitator of Cherenkov light pulses from high energy showers.

The LED should withstand high current pulses with up to 1 microsecond width. It is possible even to increase the light yield of the source using two or even more consecutive avalanche transistors [8].

The light pulses of the light source have rectangular shape as shown in Fig. 8. The rise and fall time of light pulses are $\leq 1 \mathrm{~ns}$, their width is $\sim 160 \mathrm{~ns}$ with 16 meters long coaxial cable. 


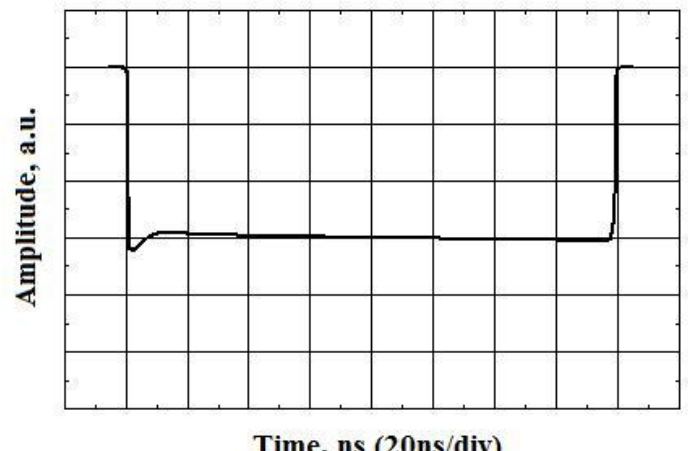

Fig. 8. Rectangular light pulses waveform of the light source imitation high energy showers.

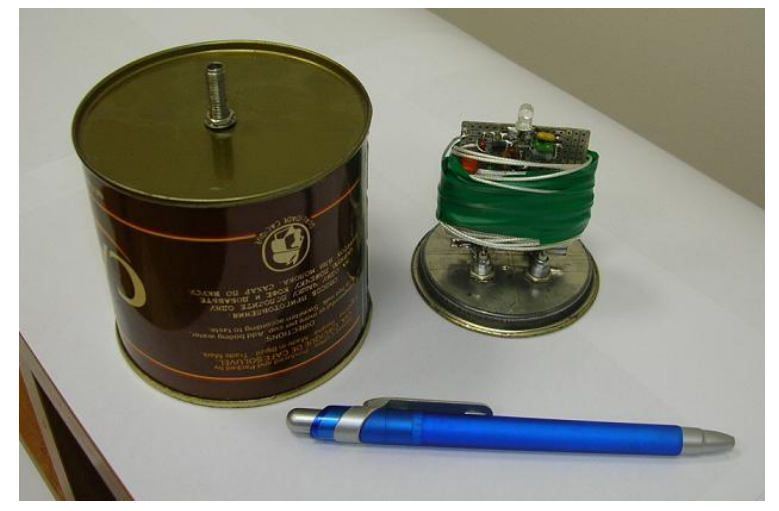

Fig. 9.

The source of rectangular shape light pulses assembled in a coffee can is shown in Fig.9. 16 meters long coaxial cable is turned around the driver's PCB board.

\section{Conclusion.}

We developed a number of light sources imitating Cherenkov and scintillation light pulses for Cherenkov and scintillation detectors used in high energy astroparticle physics experiments.

\section{Acknowledgements.}

The work was supported by the Russian Science Foundation grant no. 17-12-01331.

\section{References}

[1] Ye.E. Vyatchin. PhD Thesis. Moscow, INR 2006.

[2] Ye.E. Vyatchin, B.K. Lubsandorzhiev. Instr. and Exp. Tech. 2004. N.4. P.80.

[3] J.S. Kapustinsky et al. NIMA. 1985. V.241. P.612.

[4] B.K. Lubsandorzhiev, Ye.E. Vyatchin. JINST. 2006. 1. T06001.

[5] B.K. Lubsandorzhiev et al. Proceeding of $30^{\text {th }}$ ICRC. Vol.5. P.1117.

[6] R.V. Vasiliev, B.K. Lubsandorzhiev, P.G. Pokhil. Instr. and Exp. Tech. 2000. N.4. P.148.

[7] B.K. Lubsandorzhiev et al. NIMA. 2000. V.442. P.452. 